\title{
1Q18—the risk-capital roll continues
}

\section{Laura DeFrancesco}

The biotech sector took in record amounts of venture capital last quarter-the largest amount of venture capital in a single quarter since data provider BioCentury started tracking it in 1994. Partnerships, debt and follow-on offerings were also all up, whereas funding from

\section{Stock market performance}

Volatility in the biotech sector followed trends in stocks generally.

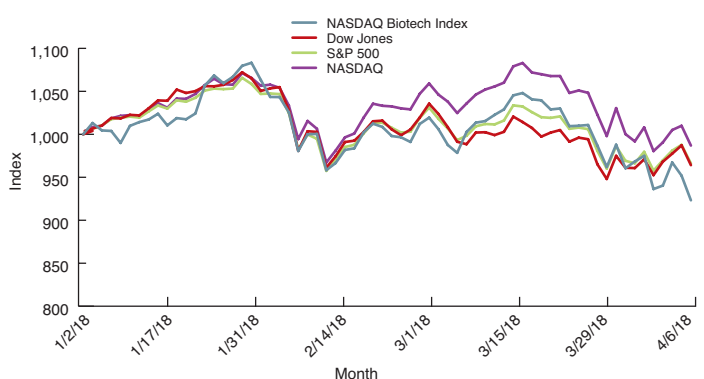

\section{Global biotech initial public offerings}

IPO funding was down globally, especially (over 70\%) in Europe and Asia.

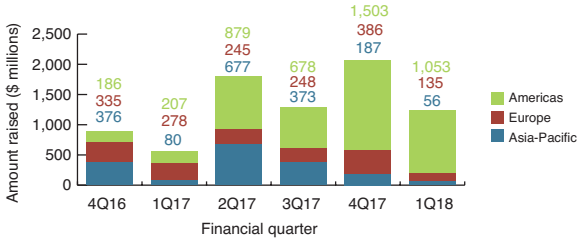

Number of IPOs

\begin{tabular}{lllllll}
\hline & $\mathbf{4 Q 1 6}$ & $\mathbf{1 Q 1 7}$ & $\mathbf{2 Q 1 7}$ & $\mathbf{3 Q 1 7}$ & $\mathbf{4 Q 1 7}$ & $\mathbf{1 Q 1 8}$ \\
\hline Asia-Pacific & 7 & 4 & 5 & 5 & 3 & 4 \\
Europe & 5 & 8 & 7 & 5 & 6 & 2 \\
Americas & 3 & 3 & 10 & 8 & 12 & 11 \\
\hline
\end{tabular}

Source: $\mathrm{BCIQ}$ : BioCentury Online Intelligence.

\section{Global biotech venture capital investment}

Biotech venture capital continued on its march, growing $5 \%$ globally over 4 Q17.

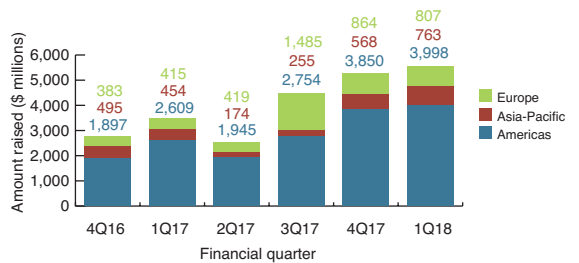

Number of rounds

\begin{tabular}{lllllll}
\hline & $\mathbf{4 Q 1 6}$ & $\mathbf{1 Q 1 7}$ & $\mathbf{2 Q 1 7}$ & $\mathbf{3 Q 1 7}$ & $\mathbf{4 Q 1 7}$ & $\mathbf{1 Q 1 8}$ \\
\hline Asia-Pacific & 10 & 10 & 11 & 8 & 14 & 14 \\
Europe & 16 & 22 & 24 & 26 & 32 & 30 \\
Americas & 51 & 85 & $\mathbf{7 3}$ & 88 & 94 & 103 \\
\hline
\end{tabular}

Source: $\mathrm{BCIQ}$ : BioCentury Online Intelligence.

\section{Global biotech industry financing}

All financing sources continued to grow, except IPOs.

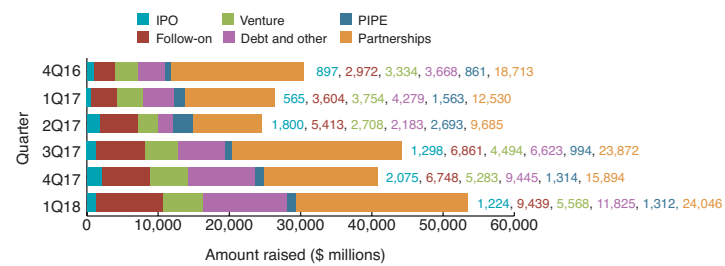

PIPEs, private investment in public equity. Source: BCIQ: BioCentury Online Intelligence. initial public offerings (IPOs) fell, especially outside the United States. Immuno-oncology continues to feature in major deals, with Celgene acquiring Juno, and Nektar signing a multibillion-dollar licensing deal with Bristol-Myers Squibb.

\section{Notable $1 Q 18$ deals}

IPOs

\begin{tabular}{|c|c|c|c|}
\hline Company (lead underwriters) & $\begin{array}{l}\text { Amount raised } \\
\text { (\$ millions) }\end{array}$ & $\begin{array}{l}\text { Date } \\
\text { completed }\end{array}$ & $\begin{array}{l}\text { Change in stock } \\
\text { price since offer } \\
\text { (as of } 5 / 4 / 2018 \text { ) }\end{array}$ \\
\hline $\begin{array}{l}\text { Homology Medicines (Cowen, BTIG, Bank of America } \\
\text { Merrill Lynch, Evercore ISI) }\end{array}$ & 166.6 & 27-Mar & $15.2 \%$ \\
\hline $\begin{array}{l}\text { Armo Biosciences (Leerink Partners, BMO Capital } \\
\text { Markets, Baird, Jeffries) }\end{array}$ & 147.2 & 25-Jan & $-5.9 \%$ \\
\hline $\begin{array}{l}\text { Solid Biosciences (JP Morgan, Goldman Sachs, Leerink } \\
\text { Partners, Nomura, Chardan Capital Markets) }\end{array}$ & 143.8 & 25-Jan & $-16.5 \%$ \\
\hline Arcus Biosciences (Goldman Sachs, Leerink Partners) & 138 & 14-Mar & $-4.2 \%$ \\
\hline $\begin{array}{l}\text { Menlo Therapeutics (JMP Securities, Jefferies, } \\
\text { Guggenheim Securities, Piper Jaffray) }\end{array}$ & 136.9 & 24-Jan & $-69.5 \%$ \\
\hline $\begin{array}{l}\text { resTOR Bio (Evercore ISI, Bank of America Merrill } \\
\text { Lynch, Leerink Partners, Wedbush) }\end{array}$ & 97.8 & 25-Jan & $-43.1 \%$ \\
\hline
\end{tabular}
Lynch, Leerink Partners, Wedbush)

Venture capital rounds

\begin{tabular}{|c|c|c|c|}
\hline Company (lead investors) & $\begin{array}{l}\text { Amount raised } \\
\text { (\$ millions) }\end{array}$ & $\begin{array}{l}\text { Round } \\
\text { number }\end{array}$ & $\begin{array}{l}\text { Date } \\
\text { closed }\end{array}$ \\
\hline $\begin{array}{l}\text { Moderna Therapeutics (Fidelity, Julius Baer, Pictet Funds, } \\
\text { Viking Global Investors and others) }\end{array}$ & 500 & Beyond $\mathrm{E}$ & $1-\mathrm{Feb}$ \\
\hline BioNTech (Invus, Fidelity, Redmile Group and others) & 270 & A & 4-Jan \\
\hline SomaLogic (Nan Fung, iCarbonX, Madryn Asset) & 200 & Not disclosed & 4-Jan \\
\hline $\begin{array}{l}\text { Oxford Nanopore (GIC-Singapore, China Construction } \\
\text { Bank, Hostplus) }\end{array}$ & 139 & Not disclosed & 20-Mar \\
\hline $\begin{array}{l}\text { TCR2 Therapeutics ( } 6 \text { Dimensions Capital, Curative } \\
\text { Ventures, Redmile Group, Arrowmark Partners and others) }\end{array}$ & 125 & B & 21-Mar \\
\hline Gossamerbio (ARCH, Omega Funds) & 100 & A & 4-Jan \\
\hline Generation Bio (Fidelity, Invus, Casdin Capital, Foresite & 100 & B & 27-Feb \\
\hline
\end{tabular}
Capital Management, Leerink Partners)

\begin{tabular}{llll} 
Mergers and acquisitions & & & \\
\hline Target & Acquirer & Value $\mathbf{\$}$ millions $)$ & Date announced \\
\hline bioverativ & Sanofi & 11,600 & 22-Jan \\
Juno & Celgene & 9,000 & 22-Jan \\
Impact Biomedicines & Celgene & 7,000 & 8-Jan \\
Ablynx & Sanofi & 4,800 & 29-Jan \\
Flatiron & Roche & 1,900 & 15-Feb
\end{tabular}

Licensing/collaboration

\begin{tabular}{|c|c|c|c|}
\hline Researcher & Investor & $\begin{array}{l}\text { Value } \\
\text { (\$ millions) }\end{array}$ & Deal description \\
\hline Eisai & Merck & 6,235 & $\begin{array}{l}\text { Eisai partners with Merck to develop and commercialize } \\
\text { worldwide Eisai's small-molecule pan-kinase vascular } \\
\text { endothelial growth factor } 1,2 \text { and } 3 \text { inhibitor Lenvima } \\
\text { (lenvatinib) as monotherapy and in combination with } \\
\text { Merck's PD-1 inhibitor Keytruda (pembrolizumab) }\end{array}$ \\
\hline $\begin{array}{l}\text { Nektar } \\
\text { Therapeutics }\end{array}$ & $\begin{array}{l}\text { Bristol-Myers } \\
\text { Squibb (BMS) }\end{array}$ & 3,650 & $\begin{array}{l}\text { Nektar Therapeutics partners with BMS to develop and } \\
\text { commercialize Nektar's PEGylated engineered inter- } \\
\text { leukin-2 (NKTR-214) in combination with BMS's PD1 } \\
\text { inhibitor Opdivo (nivolumab) and Opdivo plus CTLA-4 } \\
\text { inhibitor Yervoy (ipilimumab) in select solid tumors }\end{array}$ \\
\hline Sangamo & $\begin{array}{l}\text { Gilead } \\
\text { Pharmaceuticals }\end{array}$ & 3,150 & $\begin{array}{l}\text { Sangamo partners with Gilead's Kite Pharma to use } \\
\text { Sangamo's zinc finger nuclease and adeno-associated } \\
\text { virus technology to develop next-generation autologous } \\
\text { and allogeneic cell therapies to treat cancer }\end{array}$ \\
\hline $\begin{array}{l}\text { Ionis } \\
\text { Pharmaceuticals }\end{array}$ & $\begin{array}{l}\text { Akcea } \\
\text { Therapeutics }\end{array}$ & 1,740 & $\begin{array}{l}\text { lonis grants its subsidiary Akcea exclusive worldwide } \\
\text { rights to } 2^{\prime}-0-(2 \text {-methoxyethyl)-modified antisense oligo } \\
\text { inotersen to treat hereditary transthyretin amylodosis }\end{array}$ \\
\hline $\begin{array}{l}\text { Pieris } \\
\text { Pharmaceuticals }\end{array}$ & Seattle Genetics & 1,230 & $\begin{array}{l}\text { Seattle Genetics partners with Pieris Pharmaceuticals } \\
\text { to develop undisclosed bispecific mAb drug conjugates } \\
\text { against solid tumors and blood cancers }\end{array}$ \\
\hline $\begin{array}{l}\text { Denali } \\
\text { Therapeutics }\end{array}$ & Takeda & 1,173 & $\begin{array}{l}\text { Denali Therapeutics grants Takeda options to co- } \\
\text { develop/co-commercialize a bispecific mAb containing } \\
\text { a transferrin receptor binding region within the constant } \\
\text { region and CDRs specific for BACE1 and Tau, another } \\
\text { transferrin-binding mAb against TREM2 and an undis- } \\
\text { closed discovery-stage program }\end{array}$ \\
\hline $\begin{array}{l}\text { Theravance } \\
\text { Biopharma }\end{array}$ & $\begin{array}{l}\text { Johnson \& } \\
\text { Johnson }\end{array}$ & 1,000 & $\begin{array}{l}\text { Theravance partners with Janssen, a Johnson \& } \\
\text { Johnson subsidiary, to co-develop and commercialize } \\
\text { Theravance's oral small-molecule pan-JAK inhibitor } \\
\text { TD-1473 and related compounds in inflammatory } \\
\text { intestinal diseases }\end{array}$ \\
\hline
\end{tabular}

Laura DeFrancesco is a Senior Editor at at Nature Biotechnology. 